\title{
Injection molding simulation with solid semi-crystalline polymer mechanical behavior for ejection analysis
}

\author{
Nikolaj MOLE ${ }^{*}$, nikolaj.mole@fs.uni-lj.si, (tel.: +386 (0)1 4771 425) \\ Kristjan KREBELJ ${ }^{1}$, kristjan.krebelj@fs.uni-lj.si \\ Boris ŠTOK ${ }^{1}$, boris.stok@fs.uni-lj.si \\ ${ }^{1}$ Faculty of Mechanical Engineering, University of Ljubljana, Aškerčeva 6, SI 1000 Ljubljana, Slovenia, EU \\ *Corresponding author
}

\begin{abstract}
Injection molded products, produced from semi-crystalline polymers may include undercut features which can introduce distortion to the shape of the product during ejection. A thermo-mechanical modeling approach for simulating these advanced ejection problems is developed. The approach is formed by combining a method for three-dimensional residual stress prediction and an advanced material model for modeling the solid visco-elastoplastic mechanical behavior. The task of this work is to assess, by analyzing a plaque-like product, the performance of the approach in the absence of the distortive ejection effects. The numerically predicted product shrinkage and mass at different packing pressure settings are compared to experimental results. The effect of packing pressure on product shrinkage and mass was reproduced by the model and the final residual stress field was found to be in accordance with the expectations. This confirms that the methodology could be used to analyze advanced ejection problems.
\end{abstract}

\section{Keywords}

injection molding

numerical simulation

residual stresses

shrinkage

ejection

\section{Acknowledgements}

This research did not receive any specific grant from funding agencies in the public, commercial, or not-forprofit sectors. 


\section{Introduction}

The motivation for this work is to develop a thermomechanical model for the analysis of the ejection stage in injection molding of semi-crystalline materials. During ejection these materials may experience considerable deformation which may introduce undesirable distortions in the final product. An example application for such a model is a production problem described by Fischer [1]. A bottle cap with an internal thread (an undercut feature) was produced more economically by simplifying the ejection stage to merely striping the part from the mold core instead of unscrewing it. In this way, the tooling costs were reduced and faster ejection shortened the production cycle. The simplification was possible because the chosen polymer was very deformable. Fisher [1] reports that the acceptable shape of the product was achieved once the mold geometry had been modified adequately to compensate for the distorting effects of ejection.

As such tool modifications cause expenses and production delays, it is desirable to have a means of numerically predicting and solving these problems already in the tool design phase. This work focuses on developing such an approach with special emphasis on the implementation of a proper material constitutive model. In the investigated case, a numerical simulation of packing and cooling is performed with an advanced solid polymer constitutive model. The computed shrinkage and mass results are then compared to the measurements from the corresponding experiments in order to validate the developed numerical model.

The remainder of this introduction consists of a brief description of the ejection stage followed by a literature background and an overview of this contribution.

\section{$1.1 \quad$ Ejection}

The ejection phase begins with the opening of the injection mold. In the product, which is at this time sticking to the mold core, a stress field has developed depending on the packing pressure and temperature field evolution, the latter governing the solidification advancement and thermal straining. Thus, before an ejection analysis could be conducted, the product's material state prior to ejection must be predicted. In this regard, for the final geometry of the ejected product to be realistically predicted, in particular when semi-crystalline products with pronounced undercut features are considered, possible non-linear material behavior should be properly accounted for. Implementing of a constitutive mechanical model capable of describing the non-linear phenomena in the packing and cooling stages is the task of this work

\subsection{Literature}

This work refers to three groups of publications: the prediction of residual stresses, modeling of the ejection stage and the constitutive modeling work of Drozdov et al. $[2,3]$. Prediction of the residual stresses in injection molded products has received a lot of attention in the literature on injection molding simulation. It is performed by modeling the thermo-mechanical state evolution of an injection molded product throughout the production cycle. The predicted thermo-mechanical state before ejection is taken as the initial condition in a possible subsequent simulation of ejection. While the ejection simulations in the literature mainly dealt with the prediction of the ejection force, this work extends the aims to predicting the distorting effects of ejection.

The residual stress predictions were initially based on the 2.5D modeling approach where the three-dimensional geometry of a product is described as a system of thin walls. This strongly reduced the computational effort while retaining broad applicability. Baaijens [4] was one of the first authors to predict the state of residual stresses in an injection molded plaque. Other authors followed with more elaborate geometry and use of alternative material models $[5,6]$.

The 2.5D approach was used later by various authors [7-9] in their models of ejection to predict the product state before ejection. Application of the approach in their analyses was feasible because they were primarily focused on the prediction of the ejection force of thin-walled products. Bataineh and Klamecki [8] simulated ejection of a high density polyethylene (HDPE) box. For the cases where the material did not develop nonlinear behavior because only small strains occurred, the ejection force was estimated by conducting a linear elastic analysis. Wang et al. [7] also reported on their experimental investigation of ejection of HDPE products. They acknowledged that their numerical analysis could not have been performed reliably because their model could not describe large deformations occurring during ejection. To make this possible, our work introduces a more advanced material model in a fully three-dimensional prediction of residual stresses.

An important contribution was published by Kamal et al. [10] who performed a residual stress prediction on a fully three-dimensional part. The part domain was divided into melt and solid with melt pressure from a preceding CFD simulation used in a solid mechanics simulation to predict the development of residual stresses. Their approach was modified by Krebelj et al. [11], by including the melt region in the computational domain and imposing volumetrically distributed material addition. This made the approach more suitable for its implementation in commercial finite element analysis software. In ejection simulation, access to commercially available methods of contact modeling is beneficial, because advanced ejection typically presents a complex contact problem. Krebelj et al. [11] also noted that their model can be further improved by implementing an advanced material model for the solid phase.

The current work makes use of a constitutive model for the solid behavior of semi-crystalline polymers which was 
proposed by Drozdov et al. [2, 3]. The model was found to be suitable because it describes many of the response modes of the semi-crystalline materials. At considerable strains, the model can predict the rate dependence of the response, the creep/relaxation and the hysteretic response to cyclic deformation, the latter being of particular importance in ejection simulation because the material typically experiences exactly one cycle of deformation: loading and unloading. In [12], the constitutive model of Drozdov et al. [2, 3] has been implemented by Krebelj et al. in order to analyze ejection (Fig. 1), but in their study the residual stresses due to packing and cooling stages were assumed zero because no packing and cooling simulation with the non-linear constitutive model had been performed. In consequence, the model was unable to predict the final shape of an injection molded product realistically.

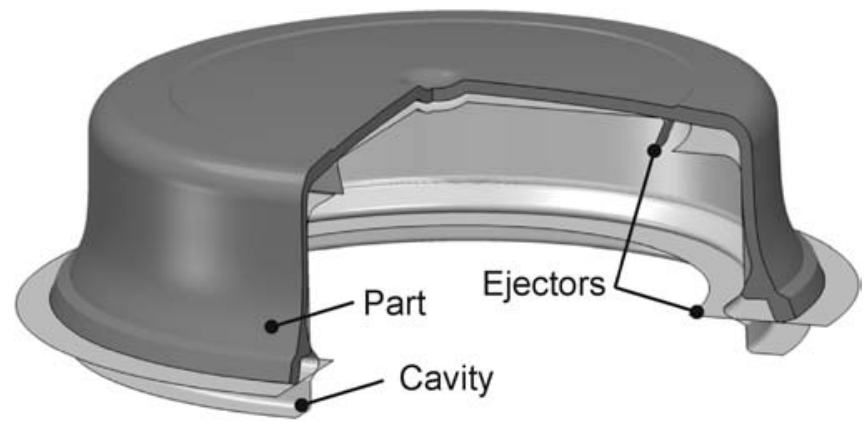

Figure 1: Example analysis of ejection of a part with undercut geometry [12]

\subsection{Overview of this work}

This work predicts the stress evolution by simulating the packing and cooling stages, release of the product from the mold and subsequent cooling to room temperature. The validity of the simulation is assessed by comparing the computed product mass and shrinkage to the existing experimental results. The geometry is elementary-a plaque.

The objectives of this work can be summarized as follows:

- $\quad$ Report the measurements of cavity pressure, mass and shrinkage for a plaque-like product at various packing pressure settings.

- Upgrade the thermo-mechanical modeling approach of Krebelj et al. [11] to include the constitutive model for solid semi-crystalline polymers of Drozdov et al. [2, 3].

- Compare the predicted product mass and shrinkage with the experimental values to obtain a thermomechanical modeling approach capable of simulating advanced ejection problems.

In Section 2 the approach of Krebelj et al. [11] is briefly reviewed. The equations for modeling the solid semicrystalline polymer mechanical response which were developed by Drozdov et al. [2, 3] are restated and the material parameter identification is described with a listing of the identified values as reported by Krebelj et al. [12].
Section 3 describes the geometry and experimental conditions for the shrinkage and mass validation. Section 4 introduces modeling concretizations to describe the experimental conditions and compares the numerical and experimental results.

\section{Modeling approach}

The modeling approach used in this paper is formed by combining two elements found in the literature: a method of predicting residual stress evolution (Krebelj et al. [11]) and an advanced constitutive model for semi-crystalline polymers (Drozdov et al. [2, 3]). The values of material parameters in the Drozdov model were determined by Krebelj et al. [12] and are in this section provided with a brief description of the characterization procedure.

\subsection{Three-dimensional stress evolution prediction}

A three-dimensional approach to predicting residual stresses was proposed by Kamal et al. [10]. To alleviate its implementation in a commercial finite element software, Krebelj et al. [11] introduced a quantity named mass factor. A brief review of the equations given in [11] follows.

The prediction of residual stresses is formulated as a problem of solid mechanics. The equilibrium condition

$\operatorname{div} \hat{\sigma}=\overrightarrow{0}$

is solved according to the finite element method. In Eq. (1), $\hat{\sigma}$ is the stress tensor and $\overrightarrow{0}$ the zero vector. The stress tensor is split into a volumetric component $-p \hat{I}$ and a deviatoric component $\hat{\tau}$

$\hat{\sigma}=-p \hat{I}+\hat{\tau}$

where $p$ is pressure and $\hat{I}$ is the identity matrix. Pressure is related to volume ratio $J$ according to an equation of state for both the melt and the solid phase with

$J=\frac{v_{s}(p, T)}{v_{s}\left(p_{0}, T_{0}\right)} f$

where $T$ is temperature, $f$ is mass factor, $v_{\mathrm{s}}(p, T)$ is specific volume and $p_{0}$ and $T_{0}$ are the initial pressure and temperature, respectively. Equation (3) was introduced by Krebelj et al. [11] to allow volumetrically distributed material addition in the melt region by increasing the mass factor $f$ from its initial value of 1 . This avoids large deviatoric deformation which is hardly tractable by the Lagrangian kinematics description used in solid mechanics simulations. During the computation the mass factor field is continuously adjusted so that the attained melt pressure approaches the melt pressure which is known from either a corresponding computational fluid dynamics (CFD) simulation or measurements, as is the case in the work of Krebelj et al. [11] and also in this contribution. The temperature field evolution can be obtained from a CFD simulation or it can be calculated along with the stresses in the solid mechanics simulation, as it is done in this work. 
The deviatoric stress $\hat{\tau}$ is phase dependent. In [11] Hooke's law was used to calculate $\hat{\tau}$ in solid polystyrene, but here the constitutive model for semi-crystalline polymers proposed by Drozdov et al. [2, 3] is adopted. The actual melt develops intractable deformations but, as already noted by Kamal [10], its key effect on the thermal residual stress state comes from pressure at solidification. This is introduced to the model by imposing the known melt pressure evolution in the melt region by means of evolving the mass factor field accordingly. To support the pressure gradient, the deviatoric strains in the melt region are assumed to develop according to Hooke's law

$\hat{\epsilon}^{d}=\frac{\hat{\imath}}{2 G_{m}}$

where $G_{\mathrm{m}}$ is the shear modulus. In this manner, a proper pressure field may develop while avoiding the development of intractable deformations. It should be stressed that Hooke's law is used as a modeling measure and $G_{\mathrm{m}}$ is not a property of the actual melt [11]. The modeling of the actual melt deformation is in this manner avoided and only its pressure effect on the solid phase is retained.

\subsection{Deviatoric response of the solid}

The deviatoric response of a semi-crystalline material is in this work described with the use of the constitutive model of Drozdov et al. [3] to which temperature dependence is introduced as published by Drozdov et al. [2]—as described later in this text. This version of their model was first tested by Krebelj et al. [12] in a simplified ejection analysis. This subsection summarizes the constitutive model only briefly. For deeper insight, the reader is advised to consult the publications of Drozdov et al. [2, 3].

The deviatoric strain tensor $\hat{\epsilon}^{\mathrm{d}}$ is split into an elastic part $\hat{\epsilon}_{\mathrm{e}}$ and a plastic part $\hat{\epsilon}_{\mathrm{p}}$ :

$\hat{\epsilon}=\hat{\epsilon}_{e}+\hat{\epsilon}_{p}$

The plastic part is likewise split into $\hat{\epsilon}_{\mathrm{pa}}$ and $\hat{\epsilon}_{\mathrm{pc}}$ corresponding respectively to the amorphous and crystalline material component. The effect of strain history is described by the dimensionless variable $\phi \in[0,1)$ governed by the differential equation

$\frac{d \phi}{d t}=a(1-\phi)^{2} e q v\left(\frac{d \hat{\epsilon}}{d t}\right)$

The initial value of $\phi$ is 0 and it increases or stagnates with deformation according to the nature of the actual loading. The dimensionless parameter $a \geq 0$ determines the rate of the accumulation of $\phi$. The equivalent deformation rate is calculated as

$e q v\left(\frac{d \hat{\epsilon}}{d t}\right)=\sqrt{\frac{2}{3} \frac{d \hat{\epsilon}}{d t}: \frac{d \hat{\epsilon}}{d t}}$

The rate of plastic deformation attributed to the crystalline component is assumed to be proportional to $\phi$ according to

$\frac{d \hat{\epsilon}_{p c}}{d t}=\phi \frac{d \hat{\epsilon}}{d t}$ while the rate of plastic deformation attributed to the amorphous component is described by

$$
\begin{aligned}
\frac{d \hat{\epsilon}_{p a}}{d t}=S\left(\hat{\epsilon}_{e}-R\right. & \hat{\epsilon}_{p a} \\
& \left.-\kappa \int_{0}^{\infty} f(v) \hat{Z}(t, v) d v\right) \text { eqv }\left(\frac{d \hat{\epsilon}}{d t}\right)
\end{aligned}
$$

where $S, R, \kappa>0$ are dimensionless parameters with their values depending on the nature of the actual loading. The integral term is the viscoelastic deformation integrated over the dimensionless energy of molecular-chain interaction $v$ on which the distribution of the viscoelastic deformation $\hat{Z}$ depends. The integral weighting function $f(v)$ is defined as

$f(v)=f_{0} \exp \left(-\frac{v^{2}}{2 \Sigma^{2}}\right)$

with $\Sigma>0$ being a material parameter and $f_{0}$ a normalization constant ensuring

$\int_{0}^{\infty} f(v) d v=1$

The time dependence of viscoelastic deformation is governed by the differential equation

$\frac{d \hat{Z}}{d t}=\Gamma(v)\left(\hat{\epsilon}_{e}-\hat{Z}(v, t)\right)$

where $\Gamma(v)$ is the rate of material creep, described by

$\Gamma(v)=\gamma \exp \left(-\frac{E_{a}}{R_{p} \theta}-v\right)$

In Eq. (13) $\gamma$ is a material parameter, $E_{\mathrm{a}}$ activation energy, $R_{\mathrm{p}}$ the universal gas constant and $\theta$ the absolute temperature [2]. The deviatoric stress is determined as

$\hat{\tau}=\left(\mu_{0}-\mu_{1} T\right)(1-\phi)\left(\hat{\epsilon}_{e}-\kappa \int_{0}^{\infty} f(v) \hat{Z}(t, v) d v\right)$

where $\mu_{0}$ is twice the shear modulus and $\mu_{1}$ a coefficient of its temperature dependence. Equations (13) and (14) which introduce temperature dependence to the model from [3] are used according to [2].

The hysteretic model response to cyclic deformation [3] is achieved by sectioning the deformation evolution in accordance with the nature of the actual loading into phases denoted by index $n$ and modifying the model parameters accordingly. Three different phases are distinguished as depicted in Fig. 2. The first loading phase is denoted by $n=1$, the unloading phase by $n=2$ and the reloading phase by $n=3$. The latter is supposed to last until the maximum equivalent strain eqv $(\hat{\epsilon})$ is achieved, from which point on the conditions characterizing the loading phase $n=1$ are reestablished. 


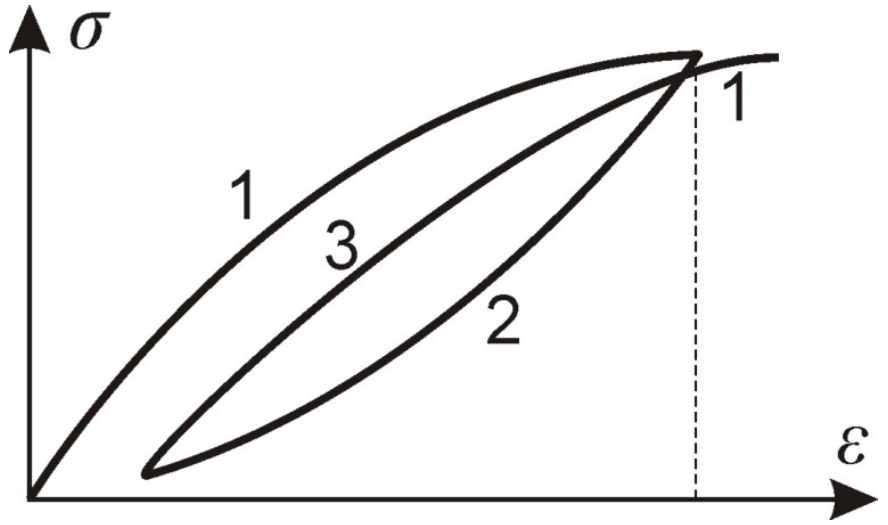

Fig. 2: Loading phases

The parameters $a, S$ and $R$ are adjusted according to the actual loading phase. The parameter $a$ assumes the values of $a_{1}>0$ and $a_{2}=a_{3}=0$, where the index corresponds to the loading phase $n$. Parameters $S$ and $R$ depend on the accumulated plastic work density

$W_{p}=\int_{0}^{t} \hat{\sigma}: \frac{d \hat{\epsilon}_{p}}{d t} d t$

The parameter $S_{n}$ is constant for $n=1$. For $n \in\{2,3\}$ the parameter $S_{n}$ is governed by the differential equation

$\frac{d S_{n}}{d W_{p}}=\beta_{n}\left(S_{n \infty}-S_{n}\right), \quad S_{n}(0)=S_{n 0}$

with $\beta_{n}, S_{n \infty}$ and $S_{n 0}$ being material constants. The parameter $R_{n}$ is for $n \in\{1,2\}$ governed by the differential equation

$\frac{d R_{n}}{d W_{p}}=\alpha_{n}\left(R_{n \infty}-R_{n}\right), \quad R_{n}(0)=R_{n 0}$

and for $n=3$ by the equation

$R_{3}=\max \left(R_{30}-R_{31} W_{p}, \quad R_{32}+R_{33} W_{p}\right)$

with $R_{30}, R_{31}, R_{32}$ and $R_{33}$ as the material constants.

\subsection{Combination of melt and solid behavior}

The material behavior was implemented by coding a FORTRAN user subroutine UMAT-Abaqus/Standard functionality to introduce a custom material model. In this procedure the stress tensor (2) was computed as a function of temperature and deformation history. Pressure $p$ was calculated from Eq. (3). The deviatoric stress $\hat{\tau}$ was expressed from Eq. (4) for the melt and from Eq. (14) for the solid, following the scheme depicted in Fig. 3.

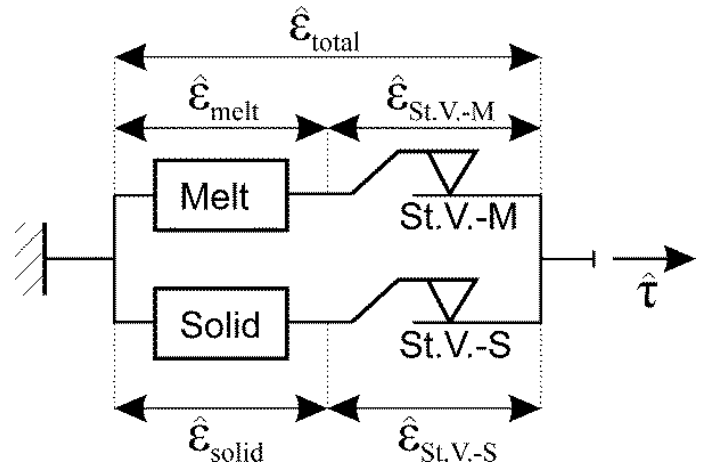

Fig. 3: Rheological model composition for the deviatoric stress

The melt and solid models were coupled in parallel to transit from melt behavior to solid behavior on solidification. At high temperatures the Saint Venant element for the melt (St.V.-M) sticks-loading the melt constitutive model-and the Saint Venant element for the solid (St.V.-S) slideseffectively suppressing the solid response. On solidification, the Saint Venant elements switch their responses, causing the newly occuring deformation to excite the solid behavior and supress the melt behavior.

2.4 Material parameters' determination and its validation Drozdov and his colleagues formulated the constitutive model by considering the material response of a series of experiments performed for that purpose. In [2, 13, 14], Drozdov et al. published the results of tensile tests, performed on HDPE with the trade name Eraclene MM 95. The tests investigated temperature dependence, rate dependence, relaxation behavior and cyclic behavior (see Figs. 4-8). These results were used by Krebelj et al. [12] to determine the material parameters of the Drozdov-model. This was done by formulating a corresponding optimization problem, where the parameters were iteratively adjusted to minimize the discrepancy between the experimental response and the model response.

The optimization problem is formulated by defining the cost function

$c(\boldsymbol{r})=\sum_{i}\left(\sigma\left(\epsilon_{i}, \boldsymbol{r}\right)-\sigma_{m}\left(\epsilon_{i}\right)\right)^{2}$

with $\mathbf{r}$ being the set of the material parameters, whose optimal values are to be found in the process of minimizing the cost function. $\sigma_{\mathrm{m}}\left(\epsilon_{i}\right)$ and $\sigma\left(\epsilon_{i}, \mathbf{r}\right)$ are respectively the measured and the computed value of the axial stress at deformations $\epsilon_{i}$. The latter is computed from the uniaxial constitutive law

$\sigma=\frac{3}{2}\left(\mu_{0}-\mu_{1} T\right)(1-\phi)\left(\epsilon_{e}-\kappa \int_{0}^{\infty} f(v) Z(t, v) d v\right)$

the scalar form of the constitutive equations (2) and (14) which is obtained by assuming incompressibility, as suggested by Drozdov et al. [15] for describing the tensile tests, and considering uniaxiality of the stress state. 
The parameters' identification procedure was conducted in the computational environment Wolfram Mathematica using a gradient descent method to minimize the cost function (19) [12]. The initial material parameters were chosen as listed by Drozdov et al. in [3] and with the temperature dependence parameters from Drozdov and Christiansen [2]. The characterized material parameters' values are tabulated in Table 1. For a detailed description of a stepwise optimization procedure see Drozdov et al. [15]. It is worth noting, however, that the material parameters presented in Table 1 describe the behavior of HDPE Eraclene MM95, as published in various publications which modeled these responses partially.

Table 1: Material parameters obtained by optimization [12]

\begin{tabular}{|c|c|c|}
\hline Param. & Value & Unit \\
\hline$\mu_{0}$ & 887.40 & $\mathrm{MPa}$ \\
\hline$\mu_{1}$ & 7.17 & $\mathrm{MPa} / \mathrm{K}$ \\
\hline$a_{1}$ & 7.06 & 1 \\
\hline$\kappa$ & 0.65 & 1 \\
\hline$E_{\mathrm{a}}$ & 38027 & $\mathrm{~kJ}$ \\
\hline$\gamma$ & $2.9 \cdot 10^{6}$ & $\mathrm{~s}^{-1}$ \\
\hline$\Sigma$ & 4.03 & 1 \\
\hline$S_{1}$ & 30.55 & 1 \\
\hline$S_{2 \infty}$ & 41.19 & 1 \\
\hline$S_{20}$ & 9.69 & 1 \\
\hline$\beta_{2}$ & 0.27 & $\mathrm{MPa}^{-1}$ \\
\hline$S_{30}$ & 40,82 & 1 \\
\hline
\end{tabular}

\begin{tabular}{|c|c|c|}
\hline Param. & Value & Unit \\
\hline$S_{3 \infty}$ & 0.31 & 1 \\
\hline$\beta_{3}$ & 0.25 & $\mathrm{MPa}^{-1}$ \\
\hline$R_{10}$ & 0.68 & 1 \\
\hline$R_{1 \infty}$ & 0.68 & 1 \\
\hline$\alpha_{1}$ & 1.60 & $\mathrm{MPa}^{-1}$ \\
\hline$R_{20}$ & 0.87 & 1 \\
\hline$R_{2 \infty}$ & 0.02 & 1 \\
\hline$\alpha_{2}$ & 323 & $\mathrm{MPa}^{-1}$ \\
\hline$R_{30}$ & 1.23 & 1 \\
\hline$R_{31}$ & 0.51 & $\mathrm{MPa}^{-1}$ \\
\hline$R_{32}$ & 0.70 & 1 \\
\hline$R_{33}$ & 0,05 & $\mathrm{MPa}^{-1}$ \\
\hline
\end{tabular}

In order to validate the performed material characterization, the model results with the parameters from Table 1 are compared to the experimental results of tensile tests from Drozdov et al. [2, 13, 14], which is presented in Figs. 4-8:

- The temperature dependence of the tensile response is presented in Fig. 4. The tests were performed and simulated at a nominal strain rate of $0.004 \mathrm{~s}^{-1}$.

- The rate dependence of the response is presented in Fig. 5 at temperature $T=25^{\circ} \mathrm{C}$.

- The temperature dependence of the relaxation behavior is depicted in Fig. 6 at strain $\epsilon_{0}=0.07$ achieved with a nominal strain rate $\dot{\epsilon}=0.04 \mathrm{~s}^{-1}$. The zero of the logarithmic scale marks the onset of the relaxation.

- The cyclic behavior was examined by Drozdov [13] for various temperatures and maximum strains (Fig. 7). The tests consisted of a phase of loading followed by a phase of unloading.

- $\quad$ The parameters for the phase of reloading $(n=3)$, were determined from the experiments performed by Drozdov and Christiansen [14] at room temperature and a strain rate of $0.002 \mathrm{~s}^{-1}$ (Fig. 8). Four tests consisted of phases: loading, unloading and reloading.

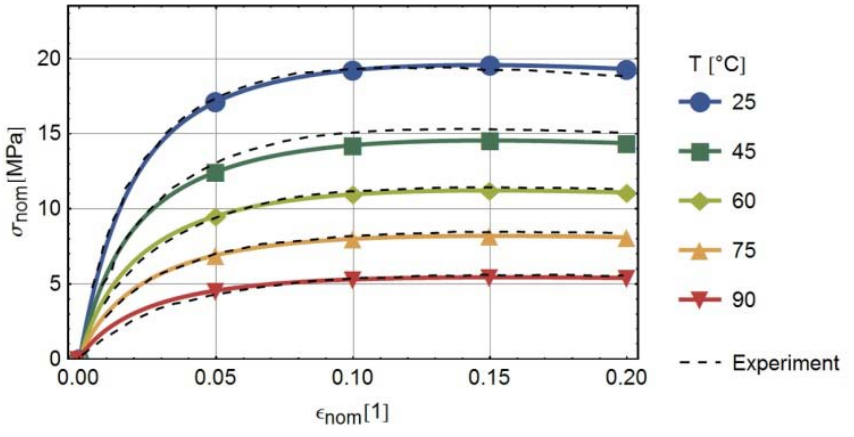

Figure 4: Uniaxial test at various temperatures [2] (strain rate: $0.004 \mathrm{~s}^{-1}$ )

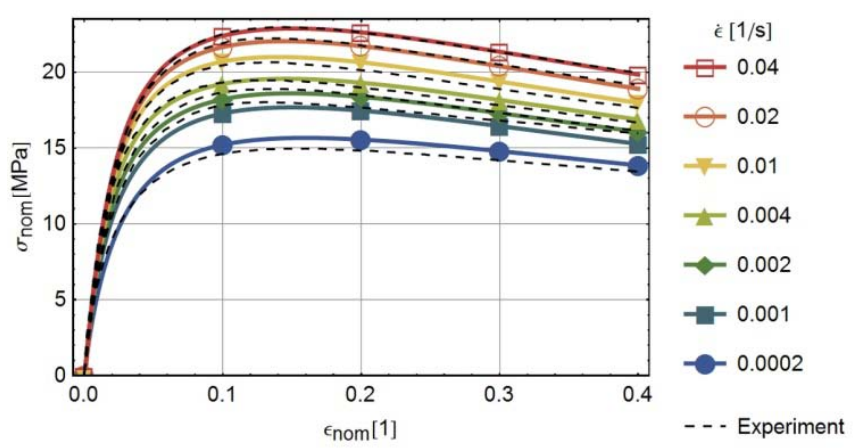

Figure 5: Uniaxial test at various strain rates [2] (temperature: $25^{\circ} \mathrm{C}$ )

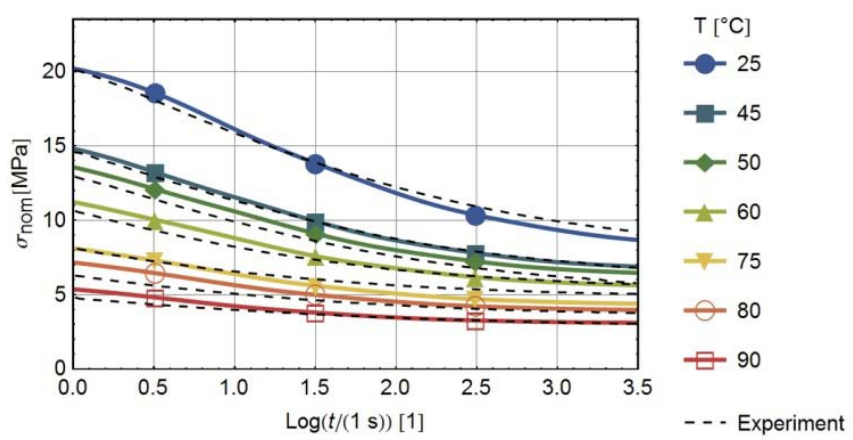

Figure 6: Relaxation dependence on temperature [2] (strain: 0.07 , achieved at the rate of $0.04 \mathrm{~s}^{-1}$ )

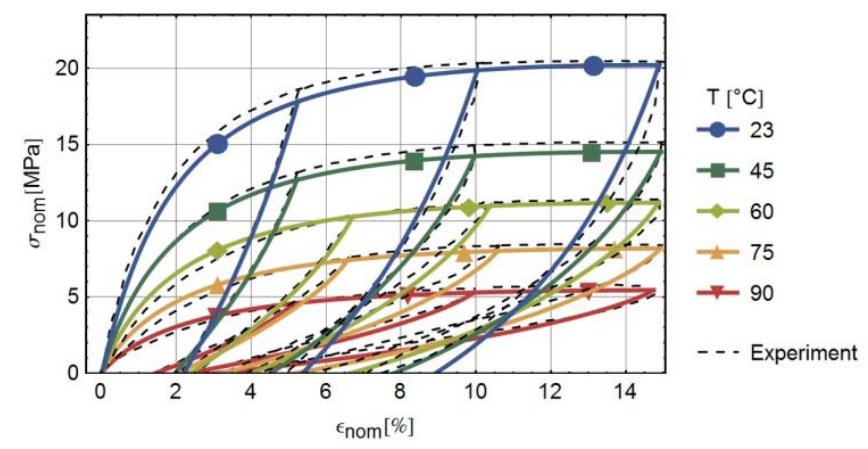

Figure 7: Loading and unloading test at various temperatures and strains unloading onset [13] (strain rate: $0.004 \mathrm{~s}^{-1}$ ) 


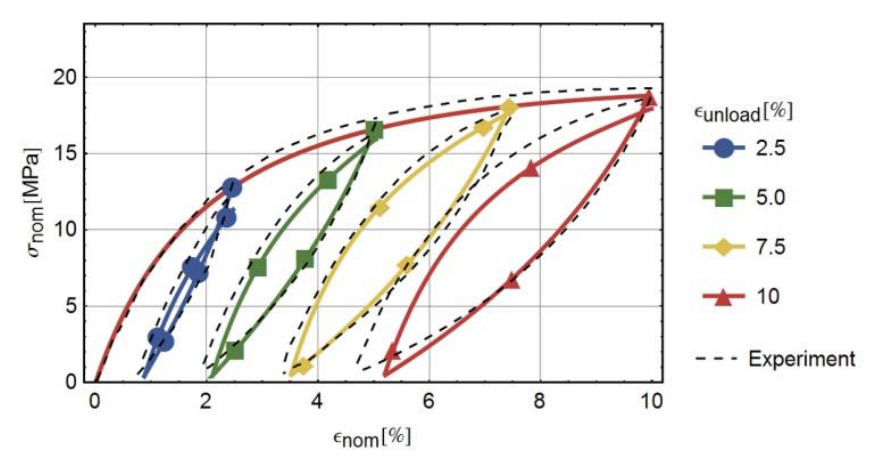

Figure 8: Cyclic behavior with reloading at various strains of unloading onset [14] (strain rate: $0.002 \mathrm{~s}^{-1}$ )

\section{Experimental case}

The conceived modeling approach was tested with a case study consisting of experimental work and a corresponding numerical model. This section presents the experimental geometry and the experimental methods which are, by providing the measured pressures that can be used later as the model input, the basis for the subsequent numerical model formulation.

\subsection{Setup and material choice}

Injection molding of plaques was performed on a Boy 50M injection molding machine with a maximum clamping force of $500 \mathrm{kN}$. As Eraclene MM95 was unobtainable, the material grade used was an HDPE with a trade name Sabic M80064. The melt temperature was set to $220{ }^{\circ} \mathrm{C}$ and the mold cooling agent (water) to $50{ }^{\circ} \mathrm{C}$. The cavity was filled in approximately $0.3 \mathrm{~s}$ with the injection rate of $10 \mathrm{~cm}^{3} / \mathrm{s}$. The packing pressure was applied for $7 \mathrm{~s}$ to ensure the gate freeze-off was completed. Further $4.5 \mathrm{~s}$ of cooling were set to precede the ejection. The packing pressure was set to eight different levels and for each level 10 specimens were collected. The experimental conditions are summarized in Table 2.

\subsection{Mold}

The experimental mold is an adaptation of a commercial mold with two cavities of which one was taken under observation. A drawing of the cavity is given in Fig 9.

Table 2: Experimental conditions

\begin{tabular}{|l|r|}
\hline Experimental setting & Value \\
\hline Melt temperature $\left[{ }^{\circ} \mathrm{C}\right]$ & 220 \\
\hline Cooling agent temper. $\left[{ }^{\circ} \mathrm{C}\right]$ & 50 \\
\hline Filling duration $[\mathrm{s}]$ & 0,3 \\
\hline Filling rate $\left[\mathrm{cm}^{3} / \mathrm{s}\right]$ & 10 \\
\hline Packing duration $[\mathrm{s}]$ & 7,0 \\
\hline Cooling duration $[\mathrm{s}]$ & 4,5 \\
\hline Packing pressure $[\mathrm{MPa}]$ & $14,18,23,29,36,45,54,64$ \\
\hline
\end{tabular}

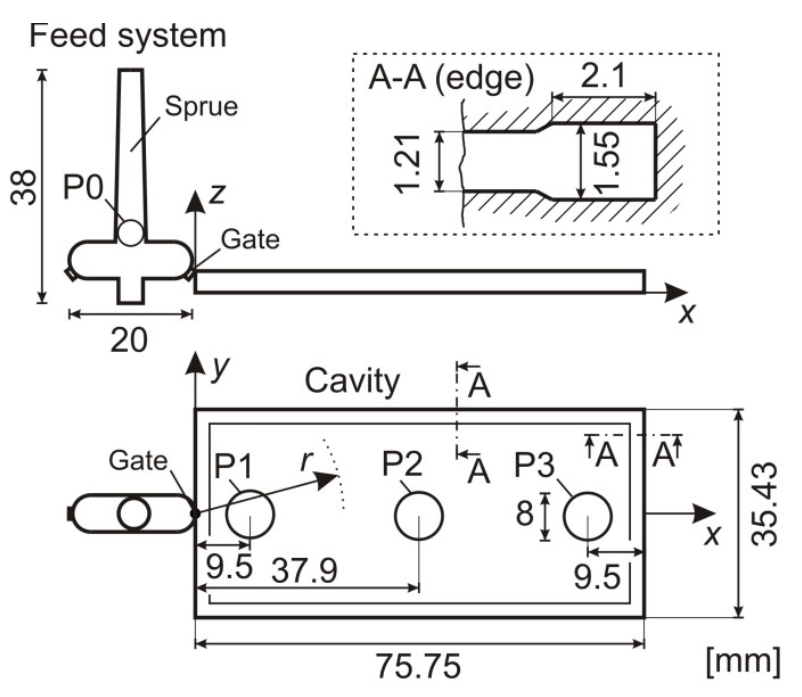

Figure 9: A drawing of the cavity under observation (as published in [11])

\subsection{Pressure measurement}

The cavity pressure was measured with a sensor of type Z1342/10000 whose signal was input to an amplifier Z134, both produced by Hasco GmbH. The sensor was positioned to 4 different measuring pins denoted by P0 to P3 in Fig. 9 and the resulting pressure measurements are given in Fig. 10. For each packing pressure setting an approximately constant value developed on the P0 position during the time interval $2 \mathrm{~s}<t<7 \mathrm{~s}$. The value was time-averaged to obtain the packing pressure level, which was used as the abscissa value when analyzing the packing pressure effect on mass and shrinkage and is numerically available in Table 2. 
(a) Setting 1, (14 MPa)

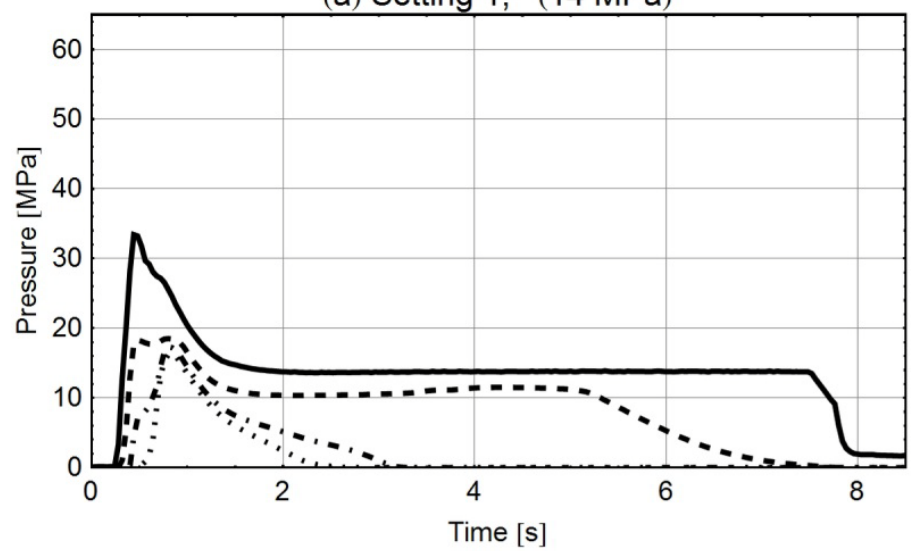

(c) Setting 3, (23 MPa)

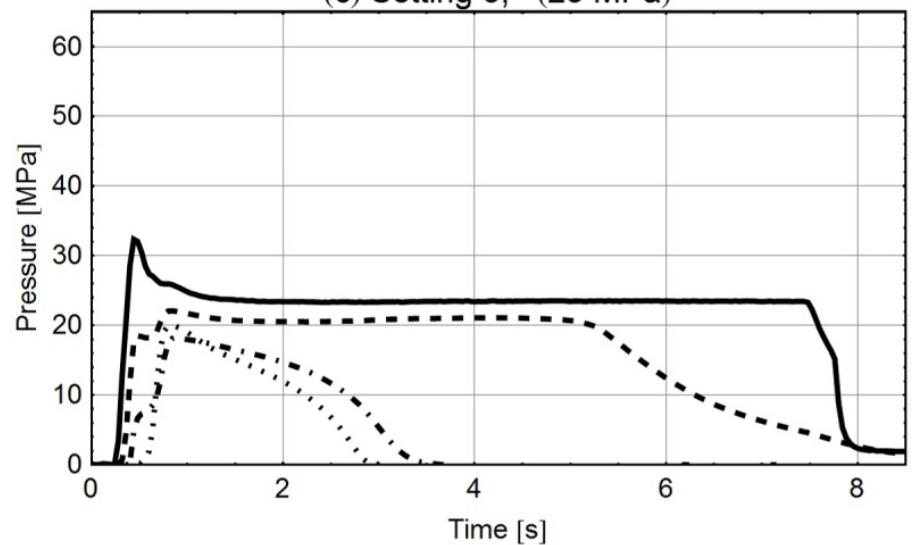

(e) Setting 5, (36 MPa)

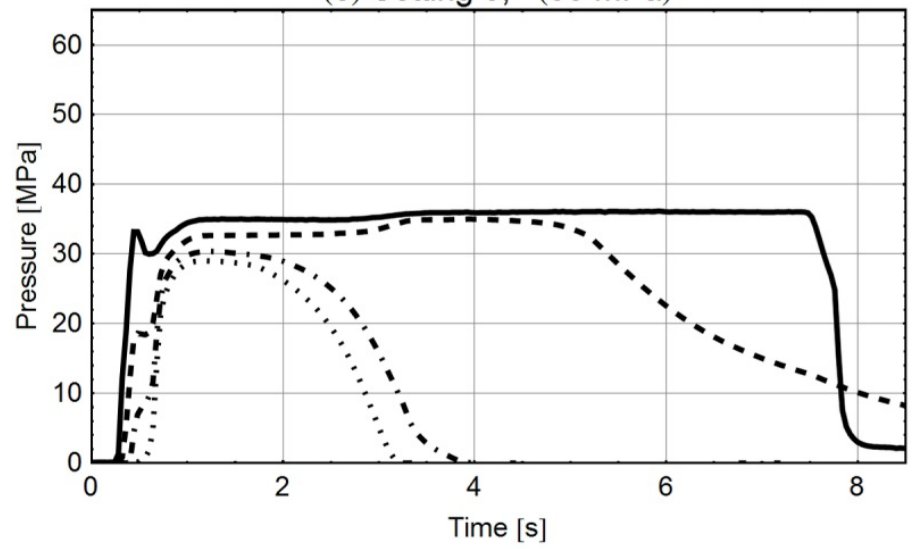

(g) Setting 7, (54 MPa)

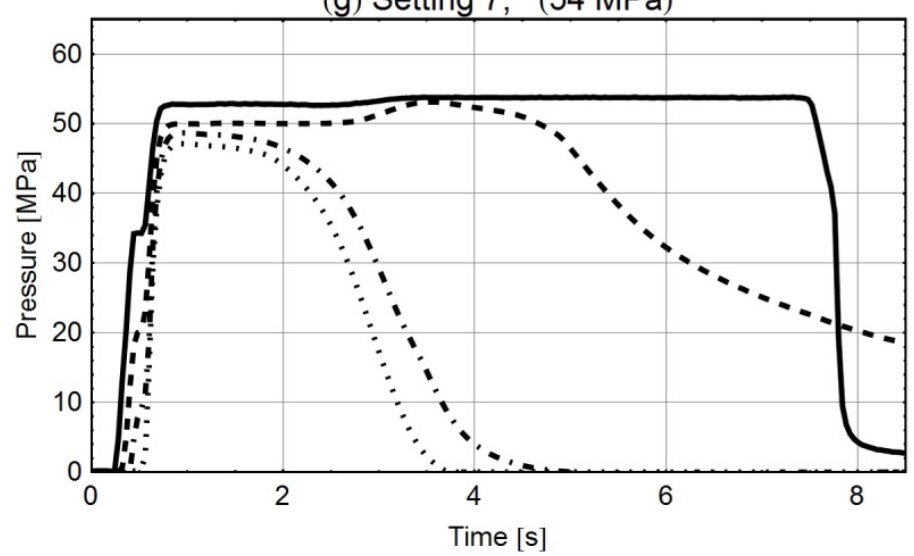

(b) Setting 2, (18 MPa)

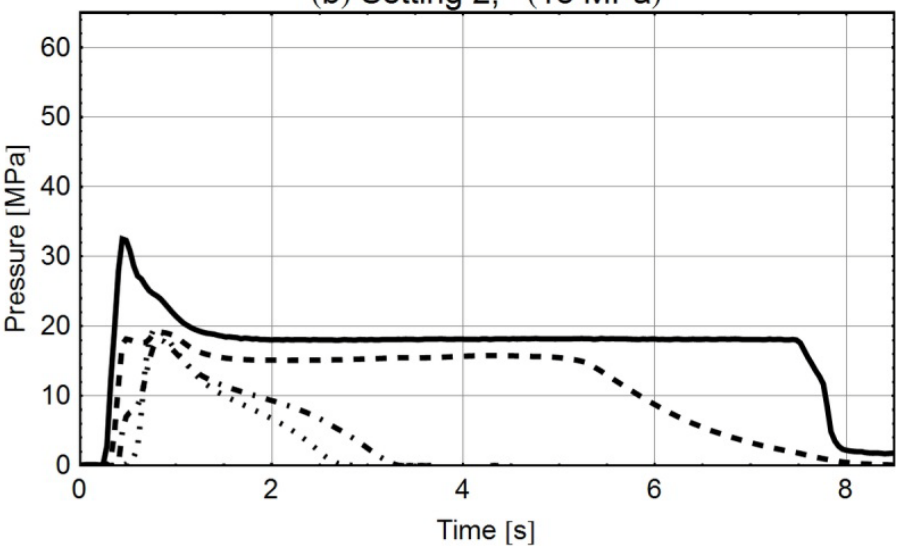

(d) Setting 4, (29 MPa)

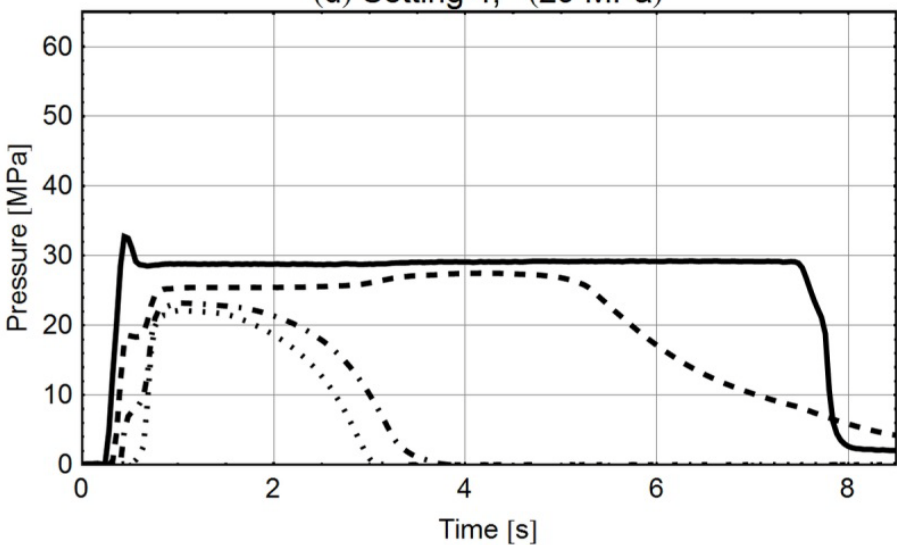

(f) Setting 6, (45 MPa)

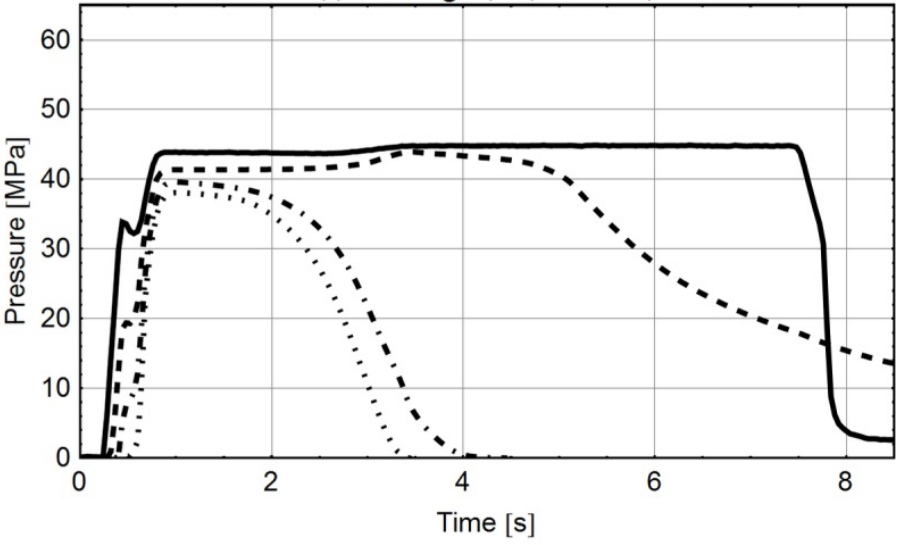

(h) Setting 8, (64 MPa)

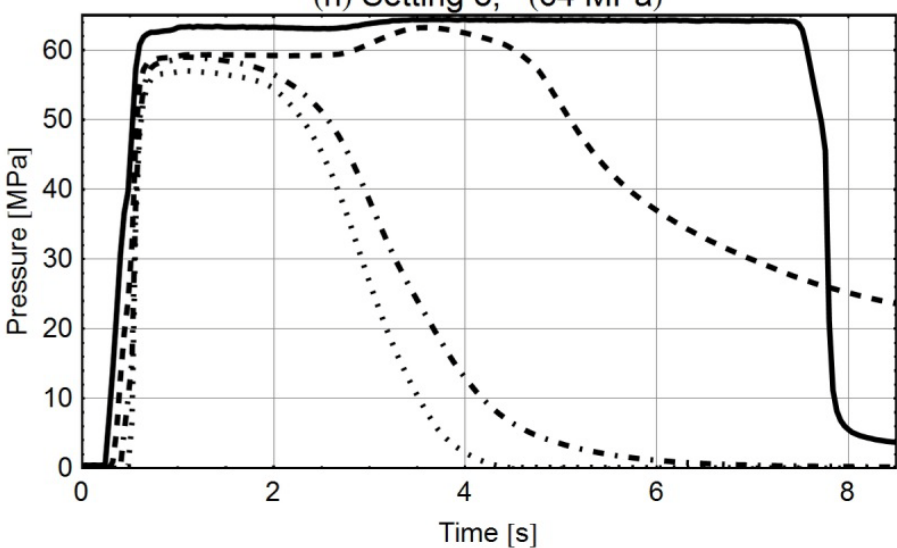

Figure 10: Measured pressure evolutions on four positions in the cavity 


\subsection{Shrinkage and mass measurement}

The produced specimens were stored for 24 hours before their length measurements were taken to assess the length shrinkage. Specimen mass was measured for 10 specimens together to obtain average values.

\section{$4 \quad$ Numerical modeling of the experimental case}

The plaque from the previous section is modeled and the computed shrinkage and mass are compared to the measured values. Geometrical simplifications were introduced to reduce the computational costs of the model. The volumetric and thermal material properties are discussed and the results are compared to experiments. This assesses the model performance in the absence of the distorting effects of ejection. The model was developed in the Abaqus/Standard simulation environment.

\subsection{Geometry and boundary conditions}

The $x z$ longitudinal cross-section captures the behavior of the whole plaque well, which is evident from the results of Krebelj et al. [11]. A large reduction in the computational effort was achieved by simplifying the domain to a thin longitudinal segment of the plaque and exploiting the symmetry over the $x y$ plane (Fig. 11).

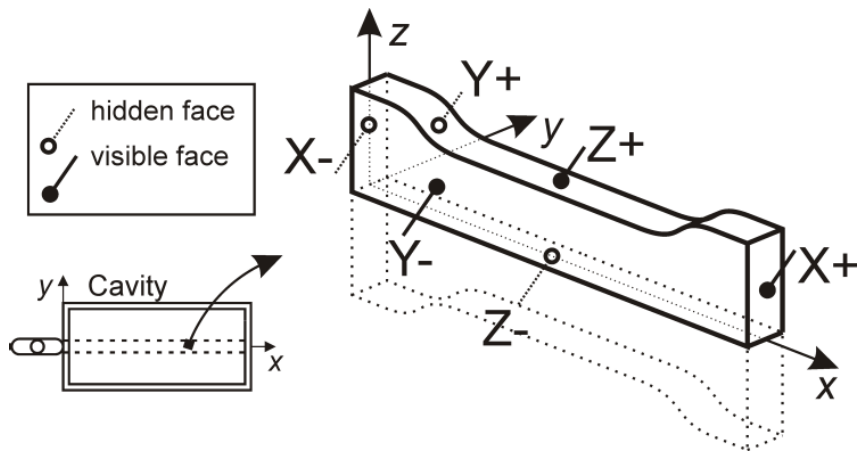

Fig. 11: A schematic representation of the model geometry

It was assumed that the physical quantities do not depend on the $y$ coordinate allowing to formulate an effectively two dimensional model (Fig. 11). The $y$ direction was treated in a similar manner as often done in the literature conducting single-dimensional (through-thickness) modeling [16, 17]. During the time the part is in the mold, the no-slip condition was assumed in the $y$ direction by imposing $\epsilon_{y}=0$ and after ejection the $\epsilon_{y}$ deformation is assumed to be constant on the whole domain.

The $\mathrm{X}+, \mathrm{X}$ - and $\mathrm{Z}+$ faces were engaged in a mechanical contact of which the $\mathrm{Z}+$ was contacting an elastic mold with a compliance

of $0.5 \mu \mathrm{m} / \mathrm{MPa}$. This value was identified by Krebelj et al. [11] for the particular experimental mold and can be considered typical [18]. The coefficient of Coulomb friction was assumed to be 0.2 .

The finite element mesh of the part consisted of 3700 hexagonal finite elements defined on 8300 nodes. At least six layers of finite elements were used in the $z$ direction (i.e. half-thickness) and one layer of elements was used in the $y$ direction. The mesh of the edge on the opposite side of the origin (i.e. injection gate) was especially refined due to higher tendency to in-mold slippage (Fig. 12).

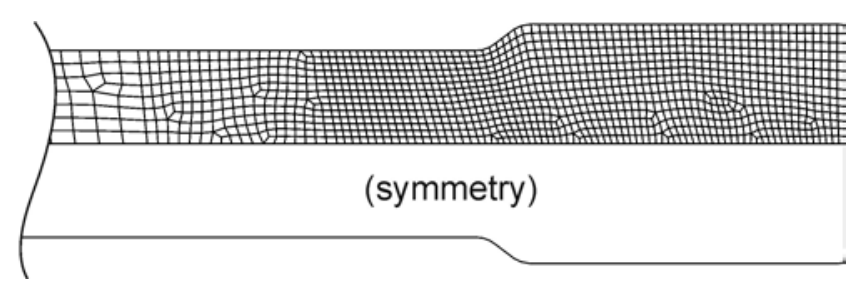

Figure 12: Mesh detail

\subsection{Volumetric material behavior}

The specific volume $v_{\mathrm{s}}$ was modeled with the use of the Tait equation of state (see Zheng et al. [19])

$v_{s}(T, p)=v_{0}(T)\left(1-C \ln \left(1+\frac{p}{B(T)}\right)\right)+v_{t}(T, p)$

The value $v_{0}(T)$ is defined as

$v_{0}(T)=\left\{\begin{array}{ll}b_{1 m}+b_{2 m}\left(T-b_{5}\right), & T>T_{t r} \\ b_{1 s}+b_{2 s}\left(T-b_{5}\right), & T \leq T_{t r}\end{array}\right.$,

where $T$ is temperature, $p$ is pressure, $T_{\mathrm{tr}}=b_{5}+b_{6} p$ is the transition temperature dividing the pressure-temperature domain into solid and melt subdomains and $b_{i j}$ are model parameters where $j$ is ' $\mathrm{m}$ ' for melt and ' $\mathrm{s}$ ' for solid. The parameter $C=0.0894$ is considered to be universal. The other functions in Eq. (21) are

$B(T)=\left\{\begin{array}{ll}b_{3 m} \exp \left(-b_{4 m}\left(T-b_{5}\right)\right), & T>T_{t r} \\ b_{3 s} \exp \left(-b_{4 s}\left(T-b_{5}\right)\right), & T \leq T_{t r}\end{array}\right.$,

and

$v_{t}(T, p)=\left\{\begin{array}{ll}0, & T>T_{t r} \\ b_{7} \exp \left(b_{8}\left(T-b_{5}\right)-b_{9} p\right), & T \leq T_{t r}\end{array}\right.$,

which are also defined piecewise on melt and solid subdomains with $b_{i j}$ being material parameters. The parameter choice is listed in Table 3 as obtained from the Moldflow material data base for HDPE Sabic M80064. The pressure field evolution is known from the experiments (Fig. 10) and was specified in the whole volume as suggested by Krebelj et al. [11].

Table 3: Material parameters for the Tait equation

\begin{tabular}{|l||l|l|c|}
\hline & Melt & Solid & Unit \\
\hline$b_{1}$ & $12.74 \cdot 10^{-4}$ & $10.75 \cdot 10^{-4}$ & $\mathrm{~m}^{3} / \mathrm{kg}$ \\
\hline$b_{2}$ & $10.26 \cdot 10^{-7}$ & $2.077 \cdot 10^{-7}$ & $\mathrm{~m}^{3} /(\mathrm{kgK})$ \\
\hline$b_{3}$ & $0.9263 \cdot 10^{8}$ & $3.324 \cdot 10^{8}$ & $\mathrm{~Pa}$ \\
\hline$b_{4}$ & $4.941 \cdot 10^{-3}$ & $2.46 \cdot 10^{-6}$ & $\mathrm{~K}^{-1}$ \\
\hline$b_{5}$ & \multicolumn{2}{|c|}{414.5} & $\mathrm{~K}$ \\
\hline$b_{6}$ & \multicolumn{2}{|c|}{$1.543 \cdot 10^{-7}$} & $\mathrm{~K} / \mathrm{Pa}$ \\
\hline$b_{7}$ & \multicolumn{2}{|c|}{$1.872 \cdot 10^{-4}$} & $\mathrm{~m}^{3} / \mathrm{kg}$ \\
\hline$b_{8}$ & \multicolumn{2}{|c|}{0.05158} & $\mathrm{~K}^{-1}$ \\
\hline$b_{9}$ & \multicolumn{2}{|c|}{$1.023 \cdot 10^{-8}$} & $\mathrm{~Pa}^{-1}$ \\
\hline
\end{tabular}




\subsection{Deviatoric material behavior}

The shear modulus of the melt region was chosen to be $G_{\mathrm{m}}=5 \mathrm{MPa}$ which is at least an order of magnitude less than for solid HDPE, allowing the melt region to change shape as if it were filled by a fluid. The solidification was chosen to onset at $125^{\circ} \mathrm{C}$. This parameter can be considered as fitted, because it was adjusted in respect to the shrinkage results. Jansen et al. [20] for example set the temperature of solidification for HDPE to $133^{\circ} \mathrm{C}$.

The solid material characterization is only available for temperatures up to $90^{\circ} \mathrm{C}$ which means that an assumption is required for the remaining temperature range of $90^{\circ} \mathrm{C}$ to $125^{\circ} \mathrm{C}$. As a minimum assumption, the (rather compliant) behavior of the material at $90{ }^{\circ} \mathrm{C}$ was assumed for this interval (Fig. 4).

Two means of improving the model robustness were used. An artificial viscosity of 0.05 MPas was used to add deviatoric stress which damped numerical instabilities and so ensured reliable convergence of the computation. The second means was an addition of stress according to a viscoelastic Maxwell model which was applied at high deformation because the solid HDPE mechanical behavior is only described for strains up to 0.4. This value tended to be exceeded at some contact points-a local surface phenomenon. Using the Maxwell viscoelastic model with the shear modulus of $10 \mathrm{GPa}$ and a relaxation time of $0.01 \mathrm{~s}$ protected these areas from overloading while having a negligible impact on the global model behavior.

\subsection{Thermal conditions}

With the pressure field known and the temperature field calculated in the solid thermo-mechanical model, there is no need for a CFD analysis to obtain these fields. Furthermore, a CFD analysis would likely introduce additional modeling uncertainties. The equation

$\rho c_{p} \dot{\theta}-\beta \theta \dot{p}=k \Delta \theta$

was used to evolve the temperature field, where $\rho$ is density, $c_{\mathrm{p}}$ specific heat, $\beta$ volumetric thermal expansion coefficient, $\theta$ the absolute temperature, $k$ the thermal conductivity and $\Delta$ the Laplace operator (see Zheng et al. [19]). Thermal conductivity measurements were performed by Dawson et al. [21] at various pressures and the results for HDPE were used in this work (Fig. 13).

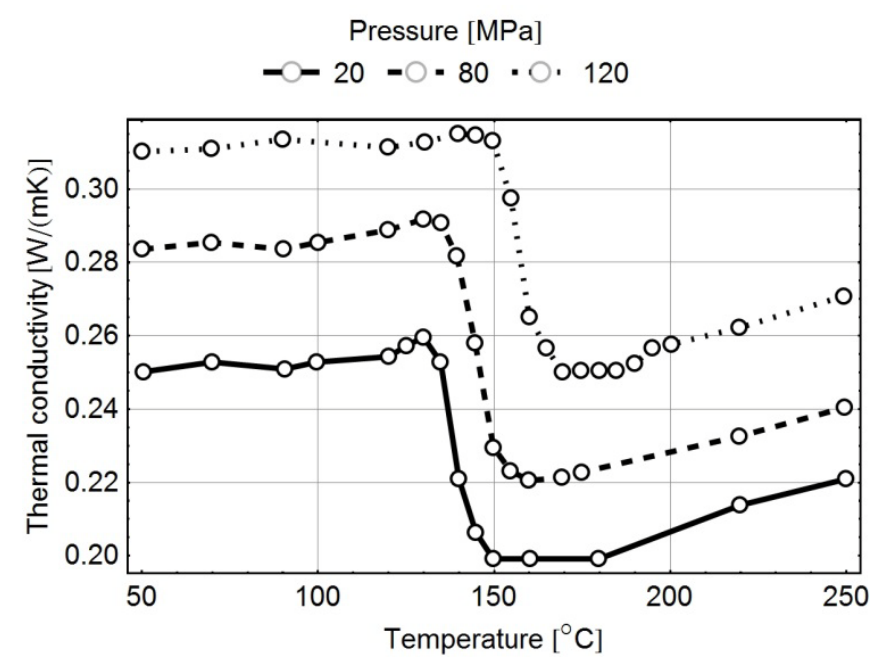

Figure 13: Pressure dependent thermal conductivity [21]

The specific heat at constant pressure $c_{\mathrm{p}}$ (Fig. 14) was calculated from the recommendations of Gaur and Wunderlich [22]. The heat of fusion was lumped into the specific heat according to the formula

$c_{p}=w_{c} c_{p}^{c}+\left(1-w_{c}\right) c_{p}^{a}-\frac{d w_{c}}{d T} \Delta H_{f}$

suggested by Gaur and Wunderlich [22], where $c_{\mathrm{p}}^{\mathrm{c}}$ is the specific heat of pure crystalline polyethylene, $c_{\mathrm{p}}^{\mathrm{a}}$ the specific heat of pure amorphous polyethylene, $\Delta H_{\mathrm{f}}$ the heat of fusion and $w_{\mathrm{c}}$ the weight fraction of the crystalline phase, which was assumed to increase from 0 to 0.66 on cooling between $150{ }^{\circ} \mathrm{C}$ and $110^{\circ} \mathrm{C}$. The resulting specific heat is compared to a temperature derivative of enthalpy measurements which were published by Mathot et al. in a collection of Brown and Gallagher [23] (Fig. 14) and the assumed specific heat is found to be a reasonable choice.

As suggested in [11], the addition of material is imposed through the control of the mass factor field only for the melt region with sufficient fluidity which was assumed to be at temperatures above $130^{\circ} \mathrm{C}$.

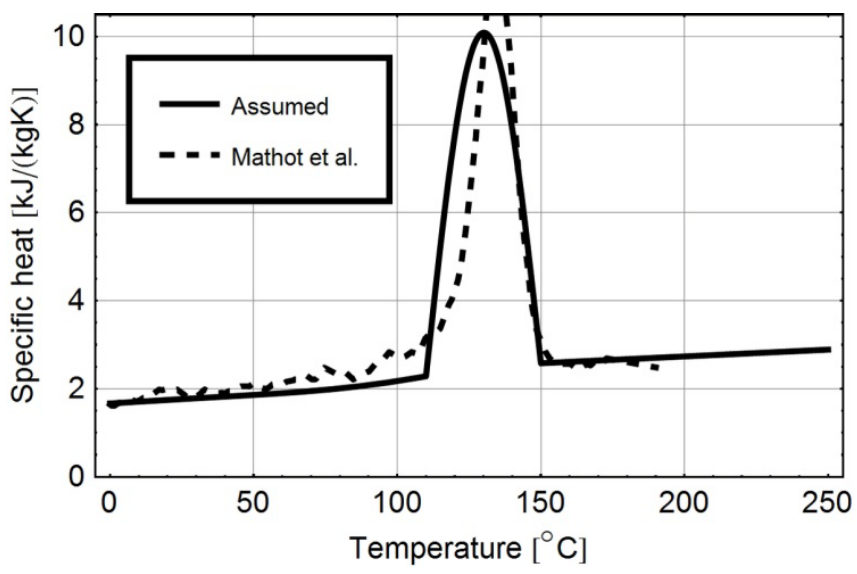

Figure 14: Specific heat for HDPE with lumped heat of fusion [22, 23]

The thermal boundary conditions were set in two ways. The surfaces $\mathrm{Y}+, \mathrm{Y}-$ and Z- (see Fig. 11) lie in the part interior and were prescribed the symmetry boundary conditions, i.e. 
were thermally isolated with the condition for heat flux $q=0$. The part exterior surfaces $\mathrm{X}+, \mathrm{X}-$ and $\mathrm{Z}+$ conduct heat to the mold during the cooling phase and are cooled by natural convection with air after ejection. The thermal boundary condition was prescribed as

$q=h_{h t c}\left(T-T_{s}\right)$

where $q$ is the heat flux directed out of the part, $h_{\mathrm{htc}}$ is the heat transfer coefficient, $T$ is the temperature at the part boundary and $T_{\mathrm{s}}$ the sink temperature. The $T_{\mathrm{s}}$ was set to 51 ${ }^{\circ} \mathrm{C}$ (as mold surface temperature) during the time the part is in the mold and as $22^{\circ} \mathrm{C}$ after the ejection. The heat transfer coefficient $h_{\mathrm{htc}}$ was set to $1.25 \mathrm{~kW} /\left(\mathrm{m}^{2} \mathrm{~K}\right)$ for the cooling inside the mold. This value was used by Krebelj et al. [11] when modeling the same experimental mold. The choice is based on the work of Yu et al. [24] who reported a measured evolution of the value between 0.83 and $2.0 \mathrm{~kW} /\left(\mathrm{m}^{2} \mathrm{~K}\right)$ and Delaunay at al. [25] who reported an evolution between 1 and $5 \mathrm{~kW} /\left(\mathrm{m}^{2} \mathrm{~K}\right)$. The cooling with air was modeled to last for $50 \mathrm{~min}$ with the choice of $h_{\mathrm{htc}}=20 \mathrm{~W} /\left(\mathrm{m}^{2} \mathrm{~K}\right)$. This value is of lesser importance and was assumed based on experience, but the choice well compares to the measurements of Koizuka and Miyanmoto [26], who determined $h_{\mathrm{htc}} \approx 4 \mathrm{~W} /\left(\mathrm{m}^{2} \mathrm{~K}\right)$ in similar conditions.

\subsection{Results}

The mass prediction is not directly available from the model, due to the geometrical modeling assumptions, i.e. the whole cavity is described by a thin layer of elements (Fig. 11). To obtain a comparable prediction, the mass of the model domain was therefore multiplied by the ratio of the full cavity volume $3416 \mathrm{~mm}^{3}$ to the model cavity volume 5.194 $\mathrm{mm}^{3}$. Fig. 15 depicts the measured and predicted part mass dependence on packing pressure where the predicted mass was about $5 \%$ higher than the measured values, which is reasonable since it is proportional to model volume as well as to the total value of the specific volume $v_{\mathrm{S}}(T, p)$. Shifting the predicted mass $5 \%$ lower reveals that the pressure dependence was captured remarkably well. Graph steepness is, on the other hand, less dependent on the particular geometry and total value of the specific volume $v_{\mathrm{s}}(T, p)$ and depends predominantly on the material compressibility and the packing phenomena which are being validated.

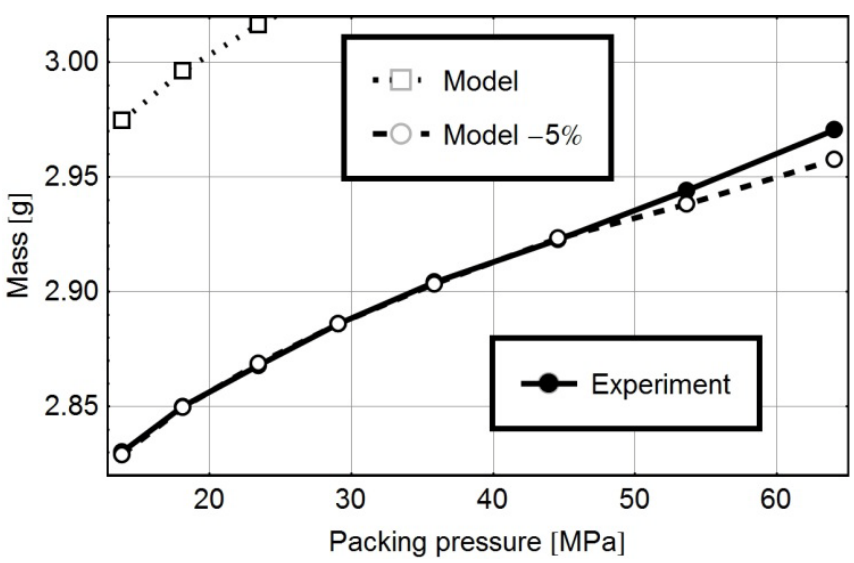

Fig. 15: Measured and predicted part mass dependence on pressure

The predicted length shrinkage is compared to the measured values in Fig. 16. The pressure dependence of the predicted graph appears to match the experimental results. The values themselves are sensitive to the assumed solidification temperature, i.e. $125{ }^{\circ} \mathrm{C}$ which is a simplification of the actual material behavior and leaves possibilities for further improvements. For comparison, a series of simulations was also run with the assumed solidification at $123^{\circ} \mathrm{C}$ and $127^{\circ} \mathrm{C}$ leading to about $0.2 \%$ different values of shrinkage for the $2{ }^{\circ} \mathrm{C}$ setting different settings. The effect on shrinkage can be understood as follows. The lower the setting, the longer material behaves as a fluid and can therefore closer match the length of the cavity, i.e. develop less length shrinkage. To illustrate, if the product hypothetically solidified and was ejected at room temperature zero shrinkage would be expected (disregarding in-mold shrinkage and the residual cavity pressure).

The experimental values at the lowest two pressure settings displayed some dispersion indicated by error bars spanning two standard deviations. Interestingly, the model behaved less consistently as well. The cause appeared to be the inmold shrinkage governed by friction and nonlinear material behavior.

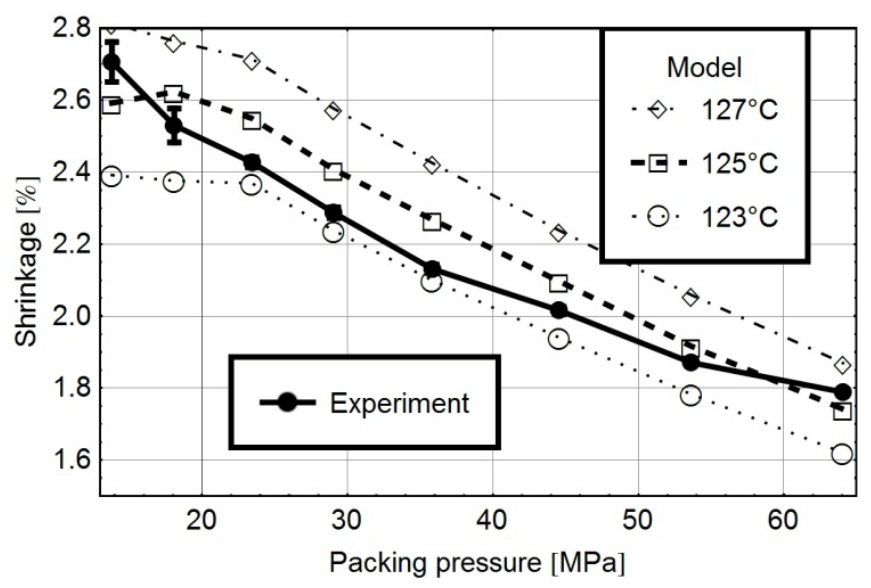

Figure 16: Measured and predicted shrinkage dependence on pressure

Figure 17 depicts the distributions of the normal components of the residual stresses in the edge region (opposite to 
injection gate) for the case with the highest and lowest packing pressures. The values of the normal (most important) components of the stress tensor serve as a strong indication of the analysis validity, because well-defined expectations exist. The $\sigma_{x}$ and $\sigma_{y}$ are the in-plane stress components and $\sigma_{z}$ is the through-thickness stress component. The in-plane components are greater than the through thickness component in most of the volume because of the wall-like geometry. The in-plane stresses are distributed as expected: tensile in the core enclosed by compressive layers-well explained by Zoetelief et. al [27]. The highest packing pressure setting caused a strongly pronounced through-thickness distribution of the in-plane residual stresses, which is typical for injection molding. Lower absolute values of these stresses occured at the lowest packing pressue setting. The magnitude of the stresses is also in accordance with the material relaxation behavior displayed in Fig. 6 which shows the stresses as high as the apparent yield stress to relax to below $10 \mathrm{MPa}$ at room temperature. The predictions may be comparatively examined against the numerical and experimental results of Kamal et al. [10], who analyzed an HDPE plaqe-like product with, unfortunately, a different experimental setup. Nevertheless, they measured residual tensile stresses up to about $10 \mathrm{MPa}$. This matches the order of magnitude of our predictions.

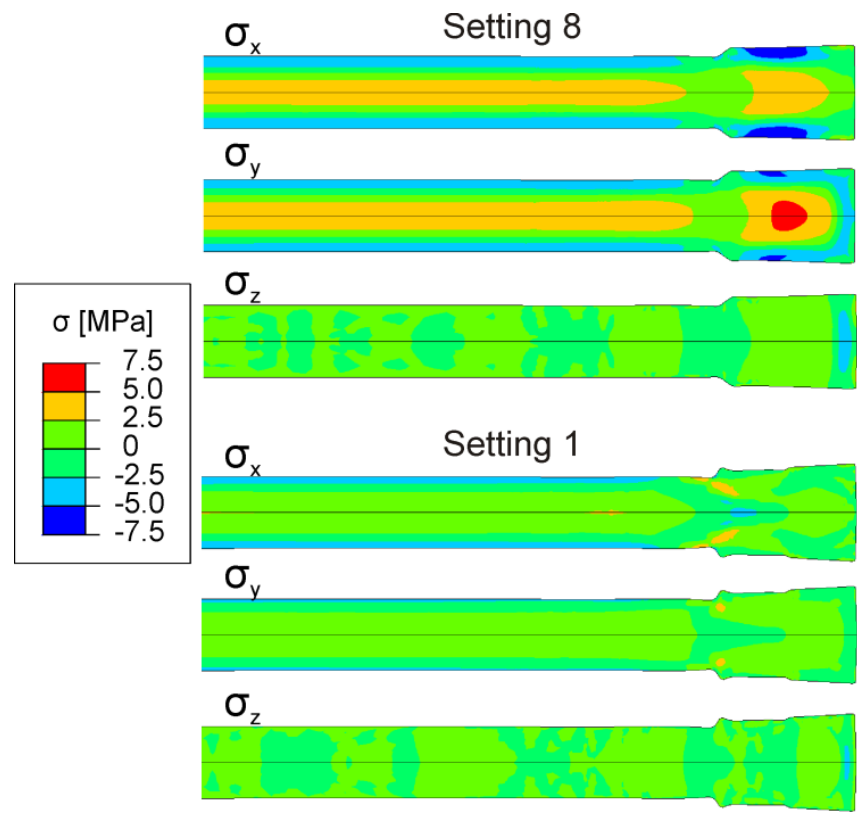

Figure 17: Normal residual stress components for the highest and lowest packing pressure settings

Fig. 18 depicts the mass factor distributions for four of eight packing pressure settings. This quantity indicates where and how much material was added in the model but should not be confused with mass density. The results indicate that more material was added in the cases with the higher packing pressure-the same finding as the progression of total mass Fig. 15. All packing pressure settings share a common quality of mass factor distribution. The part surface tends to show lower values of mass factor since it solidifies earlier which prevents mass addition. Higher mass factor values appeared in the better cooled corner areas, to compensate for the thermal material shrinkage. Lastly, the bulky wall center shows lower values of mass factor, because it solidifies at lower pressures. This corresponds to the fact that, generally, tensile stresses form in an injection molded part wall center.

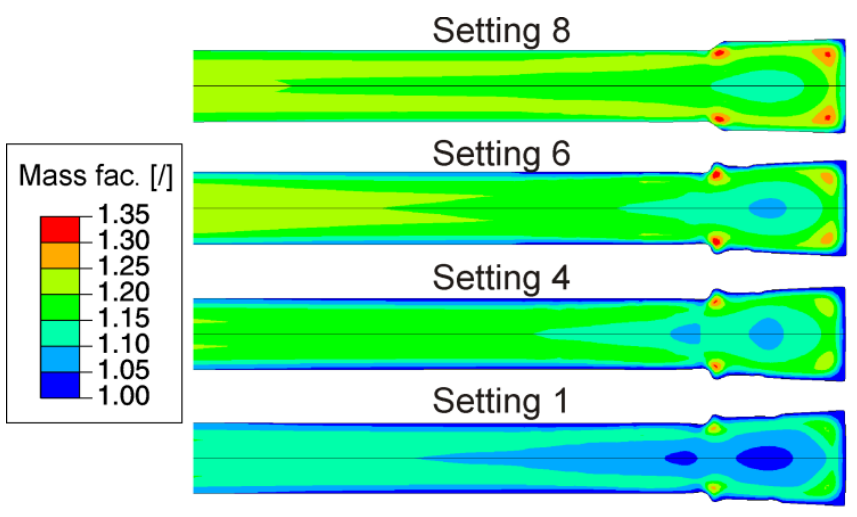

Fig. 18: Mass factor distributions for four packing pressure settings

The evolution of temperature in the wall center is displayed in Fig. 19 for the highest packing pressure setting (Setting 8) and the lowest packing pressure setting (Setting 1). At the beginning a $6{ }^{\circ} \mathrm{C}$ temperature increase in the wall center is evident at Setting 8, which is due to the compressive work and follows from the heat transfer equation (25) and reverses on decompression. A plateau is reached both on the surface and in the wall center in the temperature range between $110{ }^{\circ} \mathrm{C}$ and $150{ }^{\circ} \mathrm{C}$. This is expected due to the release of heat of fusion, which was lumped into the specific heat (Fig. 14). Measurements of wall center temperature in an HDPE disk center during cooling were performed by Kamal and Laufleuer [10] and the results display the same phenomenon-in their case at about $120^{\circ} \mathrm{C}$.

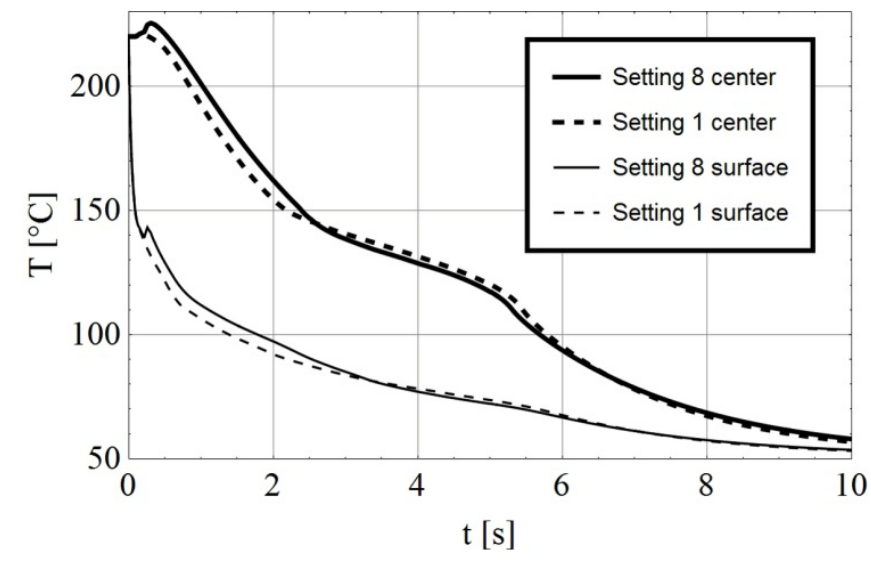

Figure 19: Temperature evolution in the plaque center and at its surface

\section{Conclusion}

By supplementing a residual stress simulation with an advanced constitutive model for solid semi-crystalline materials a model was obtained which is capable of 
describing large deformations in injection molding ejection [12]. The model was tested in the absence of the distortive ejection effects to avoid ambiguity in cause determination. Product mass and shrinkage were found to behave in a similar manner as experimentally determined. Temperature evolution in the center of the plaque and the field of normal residual stresses appeared to be in accordance to the available results from the literature.

Further experimental and numerical modeling work is still required to evaluate the performance of the approach in the case of advanced ejection problems, e.g. performing an analysis similar to the work of Krebelj et al. [12] inclusive a simulation of in-mold phenomena and experimental assessment of the final part geometry. Additionally the inclusion of a CFD predicted pressure field evolution is still lacking. This would complete the methodology of analyzing advanced ejection problems in a virtual environment.

\section{Bibliography}

1. Fischer JM (2003) Handbook of molded part shrinkage and warpage. Plastics Design Libłaikliam Andrew Pub., Norwich, N.Y.

2. Drozdov AD, Christiansen J deC. (2008) Thermo-viscoelastic and viscoplastic behavior of high-density polyethylene. Int J Solids Struct 45:4274-4288. doi: 10.1016/j.ijsolstr.2008.03.008

3. Drozdov AD, Klitkou R, Christiansen J deC. (2013) Multi-cycle deformation of semicrystalline polymers: Observations and constitutive modeling. Mech Res Commun 48:70-75. doi: 10.1016/j.mechrescom.2013.01.001

4. Baaijens FPT (1991) Calculation of residual stresses in injection molded products. Rheol Acta 30:284-299. doi: 10.1007/BF00366642

5. Chang R-Y, Chiou S-Y (1995) A unified K-BKZ model for residual stress analysis of injection molded three-dimensional thin shapes. Polym Eng Sci 35:1733-1747.

6. Kabanemi KK, Aït-Kadi A, Tanguy PA (1995) Prediction of residual flow and thermoviscoelastic stresses in injection molding. Rheol Acta 34:97-108.

7. Wang H, Kabanemi KK, Salloum G (2000) Numerical and Experimental Studies on the Ejection of Injection-Molded Plastic Products. Polym Eng Sci 40:826-840.

8. Bataineh OM, Klamecki BE (2005) Prediction of local part-mold and ejection force in injection molding. J Manuf Sci EngTrans Asme 127:598-604.

9. Pontes AJ, Pouzada AS, Pantani R, Titomanlio G (2005) Ejection force of tubular injection moldings. Part II: A prediction model. Polym Eng Sci 45:325-332. doi: 10.1002/pen.20275

10. Kamal MR, Lai-Fook RA, Hernandez-Aguilar JR (2002) Residual thermal stresses in injection moldings of thermoplastics: a theoretical and experimental study. Polym Eng Sci 42:1098-1114.

11. Krebelj K, Mole N, Štok B (2017) Three-dimensional modeling of the stress evolution in injection molded parts based on a known melt pressure field. Int J Adv Manuf Technol 90:2363-2376. doi: 10.1007/s00170-016-9533-0

12. Krebelj K, Mole N, Štok B (2016) Numerical modeling of the mechanical response of highdensity polyethylene under the circumstances of ejection in injection molding. Bovec, Slovenia (in Slovenian)

13. Drozdov AD (2010) Cyclic thermo-viscoplasticity of high density polyethylene. Int J Solids Struct 47:1592-1602. doi: 10.1016/j.ijsolstr.2010.02.021

14. Drozdov AD, Christiansen J deC. (2007) Cyclic viscoplasticity of high-density polyethylene: Experiments and modeling. Comput Mater Sci 39:465-480. doi: 10.1016/j.commatsci.2006.07.014

15. Drozdov AD (2011) Cyclic viscoelastoplasticity and low-cycle fatigue of polymer composites. Int J Solids Struct 48:20262040. doi: 10.1016/j.ijsolstr.2011.03.009

16. Bushko WC, Stokes VK (1995) Solidification of thermoviscoelastic melts. Part I: Formulation of model problem. Polym Eng Sci 35:351-364. doi: 10.1002/pen.760350409

17. Jansen KMB, Titomanlio G (1996) Effect of pressure history on shrinkage and residual stresses-injection molding with constrained shrinkage. Polym Eng Sci 36:2029-2040. 
18. Pantani R, Speranza V, Titomanlio G (2001) Relevance of mold-induced thermal boundary conditions and cavity deformation in the simulation of injection molding. Polym Eng Sci 41:2022-2035.

19. Zheng R, Tanner RI, Fan X-J (2011) Injection Molding. Springer Berlin Heidelberg, Berlin, Heidelberg

20. Jansen KMB, Van Dijk DJ, Husselman MH (1998) Effect of processing conditions on shrinkage in injection molding. Polym Eng Sci 38:838-846.

21. Dawson A, Rides M, Nottay J (2006) The effect of pressure on the thermal conductivity of polymer melts. Polym Test 25:268-275. doi: http://dx.doi.org/10.1016/j.polymertesting.2005.10.001

22. Gaur U, Wunderlich B (1981) Heat Capacity and Other Thermodynamic Properties of Linear Macromolecules. II. Polyethylene. J Phys Chem Ref Data 10:119-152.

23. Brown ME, Gallagher PK (2011) Handbook of Thermal Analysis and Calorimetry: Recent Advances, Techniques and Applications. Elsevier Science

24. Yu CJ, Sunderland JE, Poli C (1990) Thermal contact resistance in injection molding. Polym Eng Sci 30:1599-1606. doi: 10.1002/pen.760302408

25. Delaunay D, Le Bot P, Fulchiron R, et al (2000) Nature of contact between polymer and mold in injection molding. Part I: Influence of a non-perfect thermal contact. Polym Eng Sci 40:1682-1691. doi: 10.1002/pen.11300

26. Koizuka A, Miyamoto M (2005) Heat transfer from plastic film heated by thermal radiation. Heat Transfer-Asian Res 34:265-278. doi: 10.1002/htj.20059

27. Zoetelief WF, Douven LFA, Housz AJI (1996) Residual thermal stresses in injection molded products. Polym Eng Sci 36:1886-1896. doi: 10.1002/pen.10585 